\title{
Judging causal associations in observational research on caudal anesthesia and hypospadias repair
}

Sir-I was pleased to see Dr. Polaner et al.'s editorial ${ }^{1}$ accompanying Dr. Taicher et al's study. ${ }^{2}$ Using a classic paper on bias in observational research ${ }^{3}$ as a guide, Dr. Polaner's conclusions can be pushed further. Dr. Taicher's study may lack internal validity due to unequal administration of caudal anesthesia (selection bias, as they described $^{2}$ ) and the possible confounding described by Dr. Polaner. When contrasting results from these retrospective studies, selection bias will vary by local practice. These and other limitations of observational research can be enumerated by Hill's criteria for judging causal associations in observational research. ${ }^{3}$ While the temporal sequence between caudal anesthesia and fistula is appropriate and occasionally the effect size is large, as in Dr. Taicher's study, the other Hill's criteria cast serious doubt as to whether this association signifies causation. Namely, the association between fistula and caudal anesthesia is inconsistent between studies, is biologically implausible, is inconsistent with other known effects of neuraxial anesthesia, has no meaningful analogy to other conditions, and there are no randomized controlled trials supporting this relationship. It may therefore be prudent to dismiss Dr. Taicher's findings as likely spurious, due in large part to their stated selection bias. This association may merely represent an indirect effect due to confounding, where caudal anesthesia reflects higher surgical complexity and is an "innocent bystander", though this is far from clear.

Additional data and analysis from Dr. Taicher's group might provide some clarity. Analysis of their caudal administration by surgical experience, hypospadias severity, and surgical duration may identify trends in their reported selection bias. The existing regression should be studied for collinearity which may have falsely inflated the odds ratio. In addition, a new multivariate regression using distal hypospadias patients alone, potentially with surgical duration and cumulative surgeon experience as continuous rather than binary variables may better address potential confounding. While this new regression will undoubtedly be underpowered, comparing the odds ratios between regressions may suggest what is driving these results. Our study's multivariate regression showed a trend toward a negative association between caudal anesthesia and fistula ${ }^{4}$ in distal hypospadias, while others showed harm or a trend toward harm. ${ }^{2}$ These analyses should help, as would a more plausible mechanism with specific and supportive evidence.

\section{ETHICAL APPROVAL}

No ethics approval required for this work.

\section{FUNDING}

Only departmental funds were used to prepare this work.

\section{DISCLOSURES}

Dr. Haydar is a co-investigator on Dr. Polaner's multicenter prospective randomized controlled trial on this subject.

\section{ORCID}

Bishr Haydar (iD http://orcid.org/0000-0003-2709-189X

Bishr Haydar

Department of Anesthesiology, Section of Pediatric Anesthesia, University of Michigan, Ann Arbor, MI, USA

Email: bhaydar@med.umich.edu

\section{REFERENCES}

1. Polaner DM, Almenrader N, Vemulakonda V. Caudal analgesia, hypospadias, and urethrocutaneous fistula: does association mean causality? Pediatr Anesth. 2017;27:676-677.

2. Taicher BM, Routh JC, Eck JB, Ross SS, Wiener JS, Ross AK. The association between caudal anesthesia and increased risk of postoperative surgical complications in boys undergoing hypospadias repair. Pediatr Anesth. 2017;27:688-694.

3. Grimes DA, Schulz KF. Bias and causal associations in observational research. Lancet. 2002;359:248-252.

4. Zaidi RH, Casanova NF, Haydar B, Voepel-Lewis T, Wan JH. Urethrocutaneous fistula following hypospadias repair: regional anesthesia and other factors. Pediatr Anesth. 2015;25:1144-1150.

\section{Caution with the Posey ${ }^{\circledR}$ Cufflator $^{\mathrm{TM}}$ cuff pressure manometers}

Sir-Measurement of cuff pressure after placement of an endotracheal tube (ETT) is standard practice for most pediatric anesthesiologists. Low cuff pressures increase the risk of aspiration and ventilator-associated pneumonia. Elevated pressures $>25-30 \mathrm{~cm} \mathrm{H}_{2} \mathrm{O}$ exceed transcapillary pressure, potentially leading to tracheal tissue ischemia., ${ }^{1,2}$ Pediatric 